\author{
Ana Lúcia F. de Lima e Silva \\ Member, $A B C M$ \\ alfsilva@unifei.edu.br \\ Federal University of Itajubá - UNIFEI \\ Institute of Mechanical Engineering \\ 37500-903 Itajubá, MG. Brazil \\ Alice Rosa da Silva \\ calicers@hotmail.com \\ Aristeu da Silveira Neto \\ Senior Member, ABCM \\ aristeus@mecanica.ufu.br \\ Federal University of Uberlândia - UFU \\ School of Mechanical Engineering \\ 38400-902 Uberlândia, MG. Brazil
}

\section{Numerical Simulation of Two- Dimensional Complex Flows around Bluff Bodies Using the Immersed Boundary Method}

This paper presents a two-dimensional numerical simulation of flows around different bluff bodies, at Re $=100$ and 200, using the Immersed Boundary (IB) method, as a sequence of a previous work. The force density term required by the IB method is obtained with the Virtual Physical Model (VPM). Simulations were carried out for two circular cylinders of different diameter in tandem, two cylinders of the same diameter in tandem and two cylinders placed in side by side arrangement. The configurations of seven cylinders in a ' $V$ ' arrangement, for angles of $40 \mathrm{o} \leq \alpha \leq 180 \mathrm{o}$, were also simulated. A configuration of 23 different bluff bodies, representing a transverse cut in a central tower of an offshore structure, has been also simulated and the results were compared with a single compact square, of equivalent size. The Strouhal number, the drag and the lift coefficients were also calculated. The Strouhal number is calculated using the Fast Fourier Transform (FFT) of the lift coefficient temporal distribution. Visualization of the vorticity and pressure fields and the streamlines are presented for each simulation showing the flow dynamics and patterns. It was possible to verify that the IB method with $V P M$ is a powerful methodology to simulate flows in the presence of complex geometries. Keywords: immersed boundary method, virtual physical model, bluff bodies, numerical simulation, cartesian grid

\section{Introduction}

The Immersed Boundary Method was designed to solve problems in the context of biological fluid mechanics, which involve the interaction of a viscous fluid with an elastic membrane (Peskin, 1977). This methodology has been extended to solve complex engineering problems like those with immersed rigid bodies (Lima e Silva et al., 2003). The main idea of this method is to use a regular, Cartesian-Eulerian grid, to represent the total fluid domain, together with a Lagrangian grid to represent the immersed boundary. The boundary applies a singular force, generated over each Lagrangian point of the immersed surface, to the fluid and at the same time, it reacts and moves inside the fluid at the local fluid velocity. The interaction between the fluid and the boundary can be modeled by a well-chosen discrete delta function, which is an approximation to the Dirac delta function. This methodology has been applied successfully for studying the flow interference between bluff bodies and the wake formed behind them. An important feature of this methodology is the modeling and computation of the Lagrangian force, which is the force over the immersed surface that represents the body. The great advantage of this methodology when compared with other numerical methods is to simulate a fixed or a moving immersed body, with any geometric shape, using a Cartesian fixed grid (Eulerian grid). If the body moves or deforms the Lagrangian grid is responsible to represent this behavior. More details of the IB with VPM can be seen in previous work (Lima e Silva et al., 2003).

Peskin (1977) proposed to model this force field using the generalized Hooke's law for immersed elastic boundaries. A constant of elasticity has been chosen to simulate the elastic boundary. Goldstein et al. (1993) proposed to calculate the fluidsolid interfacial force using a model that depends on two constants adjusted for each simulation. The force field is chosen along a specified surface to have a magnitude and direction opposing the local flow such that the flow is brought to the same velocity as the immersed boundary. This is a kind of virtual boundary condition

Paper accepted June, 2007. Technical Editor: Francisco Ricardo Cunha. that is imposed indirectly by the force field. For unsteady viscous flows the direct calculation of the force is performed by a feedback scheme in which the velocity is used to interactively determine the desired Lagrangian force field. Many other works were developed based on the Immersed Boundary Method (Saiki and Biringen, 1996; Mohd-Yusof, 1997, Ye et al., 1999, Fadlun et al., 2000, Kim et al., 2001) and the methodology has been improved for different applications (Miller and Peskin, 2005, Griffth et al., 2006). Lima e Silva et al. (2003) presented a new proposal to model the force field over the Lagrangian grid. This model does not require any constant to be adjusted nor a special interpolation scheme near the Lagrangian cells. It has been named the Virtual Physical Model (VPM). The VPM is based on the momentum equation applied to a fluid particle located over the fluid-solid interface. The Strouhal number, the recirculation bubble length, the drag, the lift and the pressure coefficient were obtained to a single two-dimensional cylinder at moderate Reynolds numbers. The model is free of modeling constants, as well as any special procedure to recompose the discretization cells near the interface.

In the present work the Immersed Boundary Method with the Virtual Physical Model (Lima e Silva et al., 2003) were applied to simulate and analyze flows over bluff bodies. Simulations of two circular cylinders in tandem and in side by side arrangement were done. Flows over other complex arrangements of cylinders such as a ' $\mathrm{V}$ ' configuration and a composition of different geometries were simulated. The results illustrate the potential of this methodology to simulate flows over any type of complex configurations of immersed bodies.

\section{Nomenclature}

$$
\begin{aligned}
& C_{d}=\text { drag coefficient }\left(F_{d} /\left(0.5 \rho U_{\infty}^{2} d\right)\right) \text {, dimensionless } \\
& \begin{aligned}
C_{l} & =\text { lift coefficient } F_{l} /\left(0.5 \rho U_{\infty}^{2} d\right) \text {, dimensionless } \\
d & =\text { diameter of the cylinder, } m \\
D & =\text { distribution function, } m^{-2} \\
f \quad & \text { frequency of vortex shedding, } s^{-1} \\
f_{1}, f_{2} & =\text { auxiliar functions of the distribution } \\
& \text { function, dimensionless }
\end{aligned}
\end{aligned}
$$






\section{Greek Symbols}

$\Delta s=$ distance between two Lagrangian points, $\mathrm{m}$

$\Delta t=$ time step, $\mathrm{s}$

$\phi=$ general variable of the Lagrangian polynomial function

$\mu=$ dynamic viscosity, $\mathrm{kg} /(\mathrm{m} \mathrm{s})$

$\rho=$ air density, $\mathrm{kg} / \mathrm{m}^{3}$

$\varphi=$ pressure correction, $\mathrm{N} / \mathrm{m}^{2}$

\section{Subscripts}

$k$ relative to the Lagrangian points

\section{Mathematical Model: Momentum Equations and Virtual Physical Model}

A mixed Eulerian-Lagrangian formulation is used to represent the flow and the immersed boundary. A Cartesian fixed grid (Eulerian grid) describes the flow using a Finite Difference method and a Lagrangian grid, composed by a finite number of points, describes the immersed bodies. The Eulerian and the Lagrangian grids are coupled by a force field calculated at the Lagrangian points and then distributed across the Eulerian nodes in the body neighborhood.

The momentum and continuity equations for an incompressible, Newtonian viscous flow can be written as:

$$
\begin{aligned}
& \rho\left[\frac{\partial u_{i}}{\partial t}+\frac{\partial}{\partial x_{j}}\left(u_{i} u_{j}\right)\right]=-\frac{\partial p}{\partial x_{i}}+\mu \frac{\partial^{2} u_{i}}{\partial x_{j} \partial x_{j}}+f_{i} \\
& \frac{\partial u_{i}}{\partial x_{i}}=0 .
\end{aligned}
$$

The Eulerian force field is given by

$$
\boldsymbol{f}(\boldsymbol{x}, t)=\sum D\left(\boldsymbol{x}-\boldsymbol{x}_{\boldsymbol{k}}\right) \boldsymbol{F}\left(\boldsymbol{x}_{\boldsymbol{k}}, t\right) \Delta s^{2}
$$

where $D\left(\boldsymbol{x}-\boldsymbol{x}_{\boldsymbol{k}}\right)$ is the distribution function, proposed by Peskin (1977), in order to calculate the Eulerian force in a discrete form. $x_{k}$ is the coordinate vector of Lagrangian points and $\boldsymbol{x}$ is the coordinate vector of the Eulerian grid. $\boldsymbol{F}\left(\boldsymbol{x}_{\boldsymbol{k}}\right)$ is the Lagrangian force density and $\boldsymbol{f}(\boldsymbol{x})$ is the Eulerian force, which is different from zero only over the immersed boundary. Equation (3) models the interaction between the immersed boundary and the fluid flow, injecting the force field into the fluid. The distribution function, which appears in Eq.(3) can be given by:

$$
D\left(\boldsymbol{x}-\boldsymbol{x}_{\boldsymbol{k}}\right)=\frac{\left.f_{1}\left[\left(x_{k}-x_{i}\right) / h\right] f_{1}\left(y_{k}-y_{j}\right) / h\right]}{h^{2}},
$$

where

$$
f_{1}(r)=\left\{\begin{array}{l}
f_{2}(r) \text { if }\|r\|<1 \\
\frac{1}{2}-f_{2}(2-\|r\|) \text { if } 1<\|r\|<2 \\
0 \text { if }\|r\| \geq 2
\end{array}\right\},
$$

and

$$
f_{2}(r)=\frac{3-2\|r\|+\sqrt{1+4\|r\|-4\|r\|^{2}}}{8} .
$$

$\Delta s$ is the distance between the Lagrangian points, as indicated in Fig. 1. The parameter $r$ is $\left(x_{k}-x_{i}\right) / h$ or $\left(y_{k}-y_{j}\right) / h, h$ is the Eulerian grid size and $\left(x_{i}, y_{j}\right)$ are the coordinates of the Eulerian points.

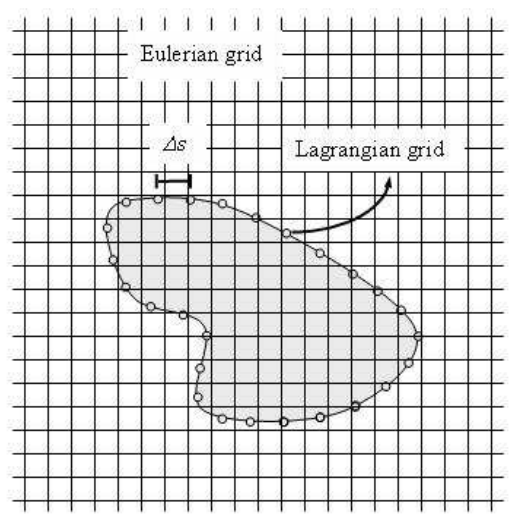

Figure 1. Immersed boundary illustration: Lagrangian grid for the interface and Eulerian grid for the domain.

The Virtual Physical Model developed by Lima e Silva et al. (2003) and used to calculate the Lagrangian force field is based only upon the momentum equations. A momentum balance is done over the fluid particle, taking into account the Lagrangian force field $\boldsymbol{F}\left(\boldsymbol{x}_{\boldsymbol{k}}, t\right)$. Isolating this Lagrangian force, yields:

$$
\boldsymbol{F}\left(x_{k}, t\right)=\boldsymbol{F}_{a}\left(x_{k}, t\right)+\boldsymbol{F}_{i}\left(x_{k}, t\right)+\boldsymbol{F}_{v}\left(x_{k}, t\right)+\boldsymbol{F}_{p}\left(x_{k}, t\right) .
$$

The different terms that compose Eq. (7) are named the acceleration force $\boldsymbol{F}_{\boldsymbol{a}}$, the inertial force $\boldsymbol{F}_{\boldsymbol{i}}$, the viscous force $\boldsymbol{F}_{\boldsymbol{v}}$ and the pressure force $\boldsymbol{F}_{\boldsymbol{p}}$. These terms are given by:

$$
\boldsymbol{F}_{\boldsymbol{a}}\left(\boldsymbol{x}_{\boldsymbol{k}}, t\right)=\rho \frac{\partial \boldsymbol{V}}{\partial t}\left(\boldsymbol{x}_{\boldsymbol{k}}\right)
$$




$$
\begin{aligned}
& \boldsymbol{F}_{\boldsymbol{i}}\left(\boldsymbol{x}_{\boldsymbol{k}}, t\right)=\rho \nabla(\boldsymbol{V} \boldsymbol{V}), \\
& \boldsymbol{F}_{\boldsymbol{v}}\left(\boldsymbol{x}_{\boldsymbol{k}}, t\right)=-\mu \nabla^{2} \boldsymbol{V}, \\
& \boldsymbol{F}_{\boldsymbol{p}}\left(\boldsymbol{x}_{\boldsymbol{k}}, t\right)=\nabla p\left(\boldsymbol{x}_{\boldsymbol{k}}\right) .
\end{aligned}
$$

The terms described by Eqs. (8)-(11) must be evaluated over the interface using the Eulerian velocity field $\boldsymbol{V}(\boldsymbol{x}, t)$ and pressure field $p(\boldsymbol{x}, t)$. These calculations also take into account that, at the interface, the fluid velocity must be equal to the interface velocity, which guarantees the no-slip boundary condition. The velocity and pressure spatial derivatives are computed using the fluid velocity and pressure obtained by means of Eqs. (1) and (2). The derivatives that compose the different terms are calculated using a second-order polynomial of Lagrange. Equations (12) and (13) were established for a generalized variable $\phi$, which can represent the velocity components and the pressure.

The derivatives in the $x$ direction are given by:

$$
\begin{aligned}
& \frac{\partial \phi}{\partial x}\left(x_{k}, y_{k}\right)=\frac{\left(x_{k}-x_{2}\right)}{\left(x_{1}-x_{2}\right)\left(x_{1}-x_{k}\right)} \phi_{1}+ \\
& \frac{\left(x_{k}-x_{1}\right)}{\left(x_{2}-x_{1}\right)\left(x_{2}-x_{k}\right)} \phi_{2}+\frac{\left(x_{k}-x_{1}\right)+\left(x_{k}-x_{2}\right)}{\left(x_{k}-x_{1}\right)\left(x_{k}-x_{2}\right)} \phi_{k}
\end{aligned}
$$

and

$$
\begin{aligned}
& \frac{\partial^{2} \phi}{\partial x^{2}}\left(x_{k}, y_{k}\right)=\frac{2 \phi_{1}}{\left(x_{1}-x_{2}\right)\left(x_{1}-x_{k}\right)}+ \\
& \frac{2 \phi_{2}}{\left(x_{2}-x_{1}\right)\left(x_{2}-x_{k}\right)}+\frac{2 \phi_{k}}{\left(x_{k}-x_{1}\right)\left(x_{k}-x_{2}\right)}
\end{aligned},
$$

with similar terms representing the $y$ direction derivatives.

The velocity components $u(x, t), v(x, t)$ and $p(\boldsymbol{x}, t)$ are interpolated over the auxiliary points $\boldsymbol{x}_{\boldsymbol{i}}$, with $i=1,2,3$ and 4 , disposed in the $x$ and $y$ direction. The velocity and pressure interpolations are performed using the neighboring Eulerian grid. After knowing the velocity components and the pressure on the auxiliary points 1, 2, 3, 4 and at point $k$, Eqs. (12) and (13) are used to evaluated each term of Eq. (7). The Lagrangian force $\boldsymbol{F}(\boldsymbol{x}, t)$ is distributed, using Eq. (3), to the Eulerian points near the interface. The scheme used for these calculations is presented in details in Lima e Silva et al. (2003).

\section{Numerical Algorithm}

Equations (1) and (2) are solved by the finite difference method through a fractional step pressure correction method (Armfield and Street, 1999). As follows, an estimation of the velocity is explicitly calculated by

$$
\frac{\bar{u}_{i}^{n+1}-u_{i}^{n}}{\Delta t}=-\frac{1}{\rho} \frac{\partial p^{n}}{\partial x_{i}}-N\left(u_{i}^{n}\right)+L\left(u_{i}^{n}\right)+f_{i}^{n}
$$

where $\bar{u}_{i}$ is the estimated velocity component, $\Delta t$ is the computational time step, $n$ is the substep index,

$$
L\left(u_{i}\right)=v \frac{\partial^{2} u_{i}}{\partial x_{j} \partial x_{j}}
$$

and

$$
N\left(u_{i}\right)=\frac{\partial\left(u_{i} u_{j}\right)}{\partial x_{j}}
$$

The pressure correction, $\varphi^{n+1}$, is obtained by solving

$$
\frac{\partial^{2} \varphi^{n+1}}{\partial x_{j} \partial x_{j}}=\frac{\rho}{\Delta t} \frac{\partial \bar{u}_{i}^{n+1}}{\partial x_{i}}
$$

The velocity field is updated using the solution of Eq. (14) by:

$$
u_{i}^{n+1}=\bar{u}_{i}^{n+1}-\Delta t \frac{\partial \varphi^{n+1}}{\partial x_{i}} .
$$

The previous pressure field and the correction pressure are used to calculate the new pressure field using

$$
p^{n+1}=p^{n}+\varphi^{n+1}
$$

The linear system for the pressure correction, Eq.(17), is solved by the modified strongly implicit procedure (MSI), Schneider and Zedan (1981). The interface force field and the momentum equations are computed using an explicit method.

A detailed description of the IB method with the VPM is presented in Lima e Silva et al. (2003).

\section{Numerical Results}

The simulations were carried out at Reynolds numbers ( $\operatorname{Re}=U_{\infty} d / \nu$ ) equal to 100 and 200, where $U_{\infty}$ is the freestream velocity and $d$ is the circular cylinder diameter or the characteristic length for the other geometries and $v$ is the kinematic viscosity of the fluid. The dimensionless time was defined as $T=t U_{\infty} / d$, where $t$ is the physical time in seconds. Once the pressure and velocities fields are obtained, the drag and lift coefficients and the Strouhal number are calculated using the Lagrangian force field, directly.

The time step of $\Delta t=1.10^{-6} \mathrm{~s}$ is used at the first iteration and is increased gradually, up to $\Delta t=1.10^{-3} s$, to ensure numerical stability. As the geometry of the interface is established by the force field, the time step is calculated in such a way that the CFL $<1.0$ criterion is attained.

A grid refinement was done with the objective of to verify its influence on the drag and lift coefficients. Different test cases varying the distance from inlet and outlet to the cylinder and the width of the domain were accomplished in order to minimize the influence of the domain on the aerodynamic coefficients. This study had also been made in the previous paper and those information were used for the present simulations. At the inlet a uniform velocity profile was imposed. At the top and bottom boundaries, the free stream condition was imposed and at the outflow the Newman boundary condition was used.

\section{Cylinders of Different Diameters in Tandem}

The IB simulations have been carried out in the domain shown in Fig. 2(a). This figure shows a global view of the domain with the main geometrical parameters. Figure 2(b) shows a closer view of the arrangement with the Cartesian and the Lagrangian grids. 
The upstream and the downstream cylinders diameters are $d$ and $d / 2$, respectively. The simulations were done for the distances between the cylinder centers of $L=1.5 d, 2.0 d, 2.5 d, 2.7 d, 3.0 d$ and $4.0 d$. A grid of $500 \times 250$ points in the $x$ and $y$ directions respectively was used. The flow direction is from the left to the right side of the domain. The simulations were carried out at a Reynolds number of 200 , based on the diameter of the upstream cylinder, and the dimensionless time step, $\Delta T=\Delta t U_{\infty} / d$, is 0.015 . The indices 1 and 2 refer to the upstream and downstream cylinder, respectively.

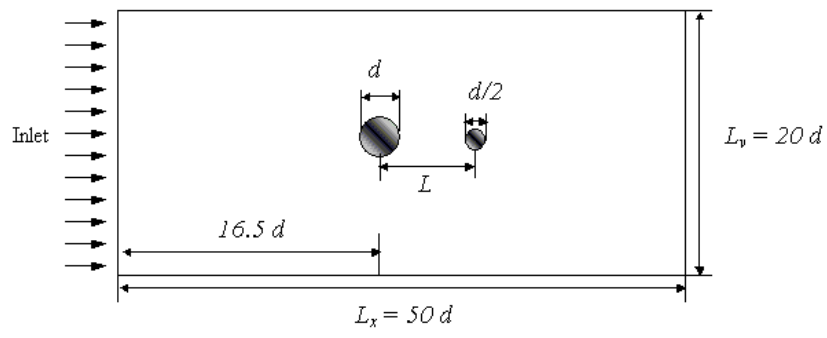

(a)

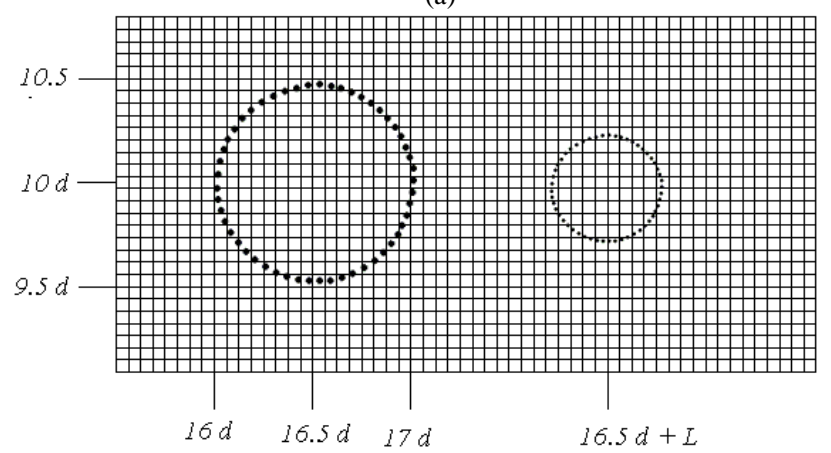

(b)

Figure 2. Geometric parameters of the simulation domain with two cylinders of different diameters disposed in tandem; global view (a) and representation of the closer view (b).

The vorticity contours for four values of the time steps are shown in Fig. 3, for $L=2.7 d$. It can be observed that, at the beginning, cylinder 2 (downwind cylinder) is embedded in the vortex street generated by the cylinder 1 . As time advances, the vortex shed by cylinder 1 retracts downward and begins to collide frontally against cylinder $2(t=10 \mathrm{~s})$. At this time, the flow near cylinder 2 changes its direction and this cylinder starts shedding vortices.

These effects (collision and vortex shedding) are directly observed in the temporal evolution of the drag coefficients as shown in Fig. 4(a). The drag coefficient of cylinder $2\left(C_{d 2}\right)$ presents negative values for $T$ up to 150 . For $T>150$, the drag coefficient assumes a positive time distribution. This behavior has its origin in the vortices transport dynamics explained above. The temporal sequence illustrated in Fig. 3 shows that as time passes the vortex shed from the upstream cylinder retracts and collides frontally with the downstream cylinder. This behavior explains why $C_{d 2}$ changes its sign as a function of time. The $C_{d 1}$ distribution also presents a sudden increase at the same time $T=150$ and also has a periodic response with time. For distances smaller than $2.7 d$ the wake formed by the cylinder 1 envelopes cylinder 2 and near its surface there is a flow in the opposite direction (from right to left).

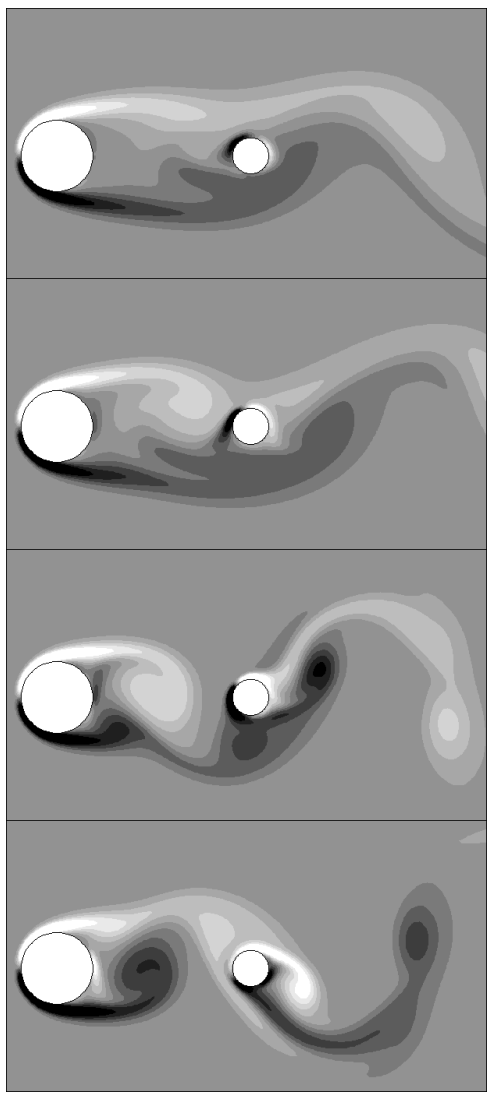

Figure 3. Arrangement in Tandem for different cylinders. Sequence of Vorticity Contours for $L=2.7 d$. From top to bottom: $t=2.5 s, 5.0 s, 10.0 s$ and $21 s$.

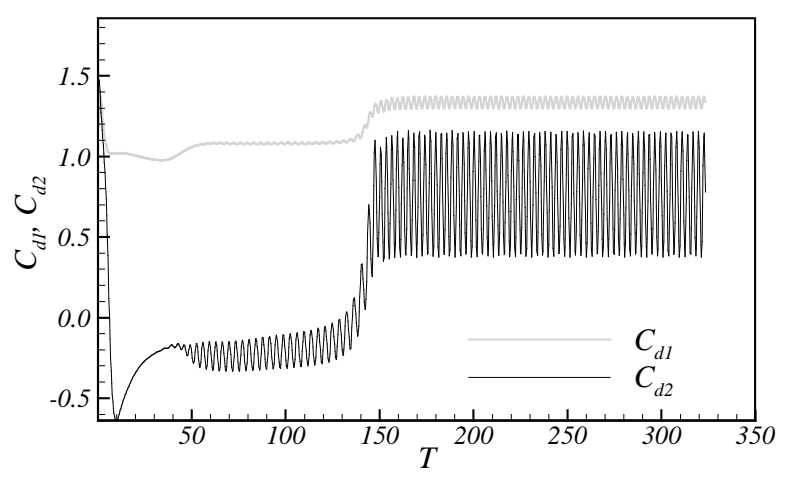

(a)



(b)

Figure 4. Arrangement in Tandem for different cylinders. Time evolution of $C_{d 1}$ and $C_{d 2}$ for $L=2.7 d(\mathrm{a})$ and the Power Spectrum (b). 
In Fig. 4(b) the power spectrum obtained with the lift coefficient of cylinder 1 is presented. There is a single dominant frequency of vortices shedding. It is important to mention that in the IB methodology, the instantaneous and the mean drag coefficient and the Strouhal number are directly obtained through the Lagrangian force, calculated over the immersed body.

The drag coefficient for both cylinders and de Strouhal number, as a function of the distance between their centers, are shown in Fig. 5. The drag coefficient sign of cylinder 2 switches from a negative value to a positive value when the vortices of cylinder 1 start to collide with cylinder 2 , as presented before. The critical value of $L / d$ $=2.7 d$ is a little larger than the value of $2.5 d$, presented by Surmas et al. (2003) and Flatschart et al. (2000). For $L / d<2.7 d$ the low pressure region formed between the cylinders induces the negative value on the downstream cylinder. The Strouhal number was calculated with the time distribution of $C_{l}$ (lift coefficient) of the upstream cylinder. In the IB method the Strouhal number can be easily obtained because the force distribution is already known. The Strouhal number was calculated by taking the Fast Fourier Transformer (FFT) of the lift coefficient distribution. The results have the same behavior when compared with other methodologies (Surmas et al., 2003 and Flatschart et al., 2000).



(a)



(b)

Figure 5. Arrangement in Tandem for different cylinders. Mean drag coefficient (a) and Strouhal number (b) as a function of the gap between cylinders centers. 1 upstream cylinder and 2 downstream cylinder.

The Strouhal number, Fig. 5(b), presents a minimum value close to the transition point (point where $C_{d 2}$ changes its sign). The alternate vortices that hit the downstream cylinder, force its shedding to occur at the same frequency of the upstream cylinder. This behavior seems to be function of the Reynolds number and of the cylinder's diameters.

\section{Cylinders of Same Diameters in Tandem}

These simulations were done for two cylinders of same diameter $d$ separated from each other by the following distances $L=1.5 d$, $2.0 d, 3.0 d$ and $4.0 d$. The simulations were carried out on a $500 \times 250$ grid for $\mathrm{Re}=200$. The domain used in these simulations is similar to the previous simulation of cylinders of distinct diameters.

Figure 6(a) shows the drag coefficient of cylinder 1 (upstream) and cylinder 2 (downstream) as a function of the distance between their centers. This figure clearly shows the point where the drag coefficient sign changes to positive. The transition occurred at $L=$ $4.0 d$ in the present work, which is the same value presented by Surmas et al. (2003) and Meneghini et al. (2001).

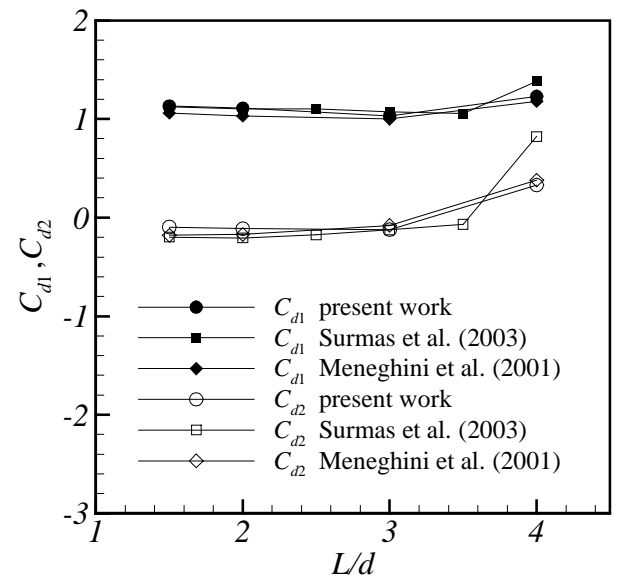

(a)



(b)

Figure 6. Arrangement in Tandem for cylinders of the same diameter. Drag coefficient for both cylinders (a) and Strouhal number (b) as a function of the gap.

When the cylinders have the same diameter, the sign transition occurs in the larger distance $(L=4.0 d)$ as compared with simulations with cylinders of different diameters $(L=2.7 d)$.

The Strouhal number, based on the diameter of cylinder 1 , is presented in Fig. 6(b) as a function of the distance and between the cylinders centers. The results of the present work are compared with results of other authors. The comparisons show that the agreement among the three methods is very good, except for $L=2.0 d$, where Surmas et al. (2003) presented a higher value. The present Strouhal value for $L=4.0 d$ was only 2 percent lower than the obtained by Meneghini et al. (2001). 


\section{Cylinders of Same Diameters Side by Side}

These simulations were done with two cylinders of same diameter disposed side by side. The gap $L$ between their centers was considered as $1.5 d, 2.0 d, 3.0 d$ and $4.0 d$. The simulation domain is similar to the presented in Fig. 2. A grid of 500x350 nodes was used and $\operatorname{Re}=200$, as in the last two cases.

Figure 7 shows the vorticity (left column) and pressure contours (right column) for all the distances simulated. Similar results of Meneghini et al. (2001) and Surmas et al. (2003) are present in the literature. There is a great interference between the wakes of the cylinders for small distances (a repulsive force between the cylinders). This repulsive force is due to the movement of the stagnation points of both cylinders where the pressure is high as observed by Meneghini et al. (2001). The flow has a region of intense instabilities that cause changes in the drag and lift coefficients. For $L \leq 2.0 \mathrm{~d}$, only one wake is formed and it deflects towards one of the cylinders and then in the opposite direction (flopping phenomenon). This behavior was also observed by Bearman and Wadcock (1973), Williamson (1985) and Meneghini et al. (2001). The formation of two independent wakes can be observed as the distance between the cylinders increases, and the wakes become independent of each other as in isolated cylinders.
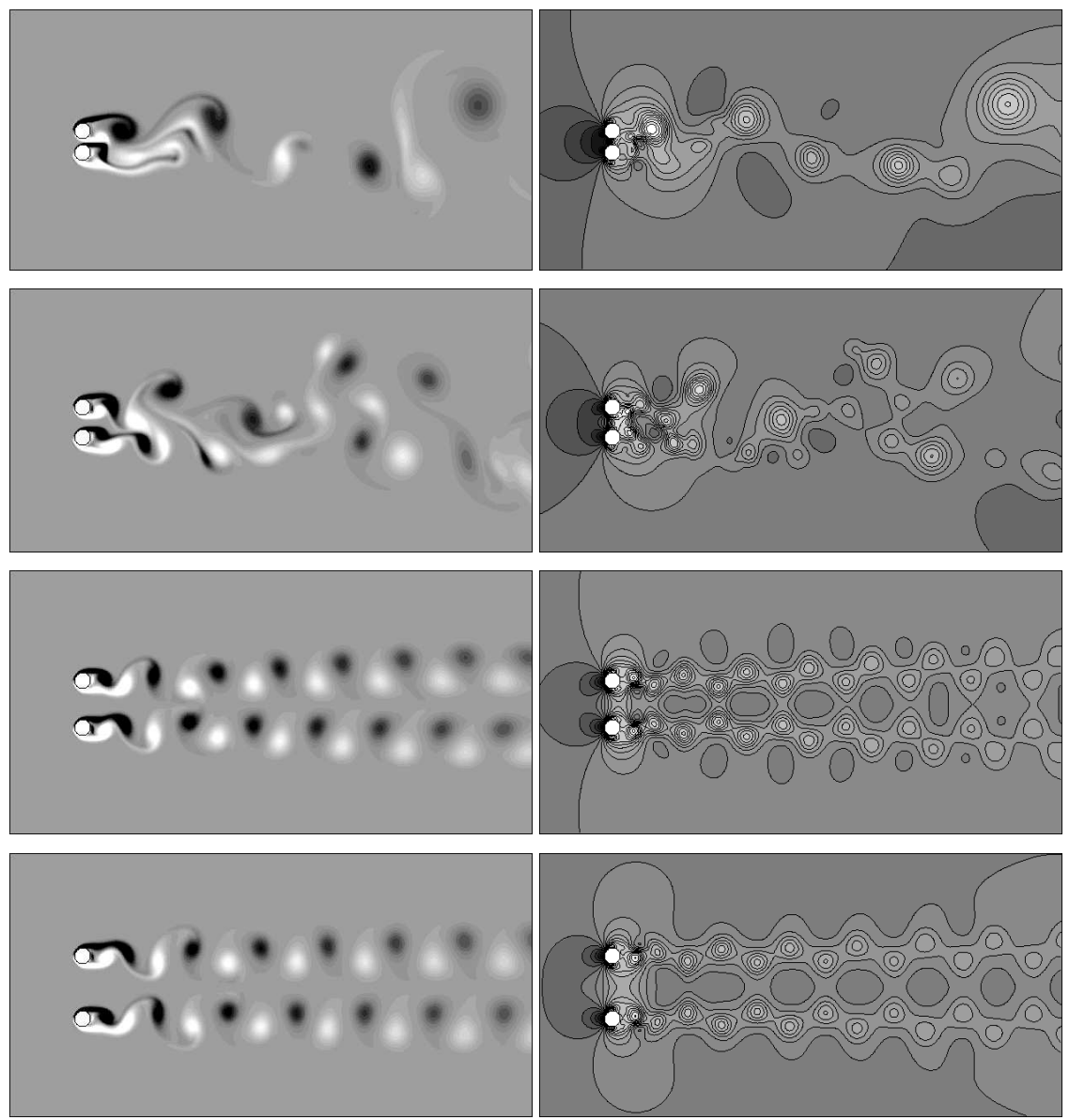

Figure 7. Vorticity Contours (left) and pressure field (right) from top to bottom, $L=1.5 d, L=2.0 d, L=3.0 d$ and $L=4.0 d$.

The mean drag and lift coefficients are presented in Fig. 8. The drag has the same value for both cylinders because they are symmetrically placed in the domain. The results of Surmas et al., (2003) and Meneghini et al. (2001) are also presented in this figure. Again, as in previous section, the agreement among the three methodologies is not good and experimental results are required in order to draw conclusions about these results.

The lift coefficient has the same magnitude for both cylinders but with opposite signs, as shown in Fig. 8(b). The index 1 indicates the upper cylinder and the index 2 indicates the lower cylinder. There is a repulsive force between the cylinders for small distances. As the gap between them increases, the mean lift coefficient for both cylinders tends to zero. This behavior was expected as it was observed for a single cylinder.

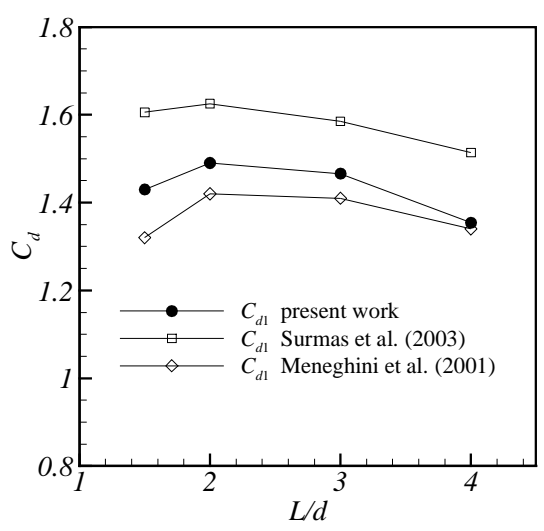

(a)

Figure 8. Drag (a) and lift (b) coefficients as a function of the gap between the cylinders for the side by side arrangement. 


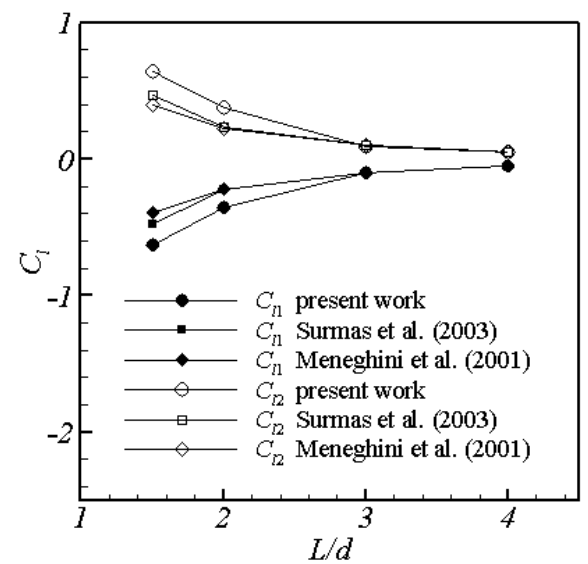

(b)

Figure 8. Drag (a) and lift (b) coefficients as a function of the gap between the cylinders for the side by side arrangement.

\section{Cylinders of Same Diameter in a ' $V$ ' Configuration}

The configuration shown in Fig. 9 was chosen to analyze and to verify the influence between the wakes formed by seven cylinders of same diameter $d$. This configuration can be related with the well known ' $\mathrm{V}$ ' formation of the birds, which in accordance with the scientists, reduces the drag force that each bird experiences compared to if it were flying alone. The cylinders were arranged in different configurations by changing the $\alpha$ angle. This angle was assumed equal to $40^{\circ}, 60^{\circ}, 80^{\circ}, 100^{\circ}, 140^{\circ}$ and $180^{\circ}$.

The diagonal distance between two consecutive cylinders, Fig. 9, was maintained equal to $2.5 d$ for all the simulations (different values of $\alpha$ ). The cylinders $1,3,5$ and 7 where chosen to be analyzed because of the flow symmetry. The Reynolds number based on the diameter of one cylinder is 100 . The grid size used for these simulations was 500x500 and the domain has dimensions $50 \mathrm{~d}$ x $50 d$.



Figure 9. Schematic view of the 'V' Configuration.

Figure 10 shows the vorticity field for all the angles simulated. It is interesting to note that the vortex formation over individual cylinders is totally inhibited when the angle $\alpha$ is small. This flow is similar to that of a single immersed body like a delta. The wake structure changes as the angle $\alpha$ increases. The flows become more complex and all the cylinders start to shed vortices, which strongly interact. There is a great influence between their wakes, which changes the drag force coefficients.



(a)



(c)

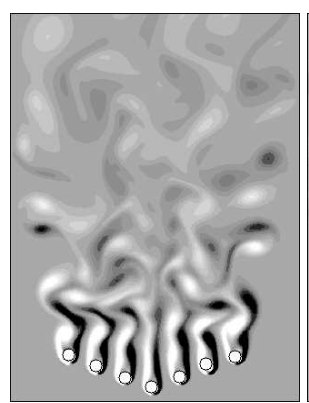

(e)

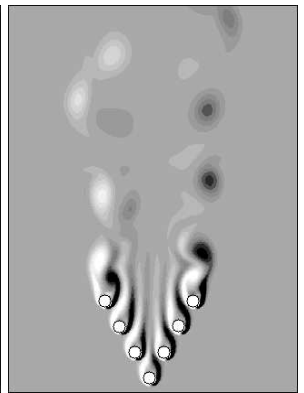

(b)

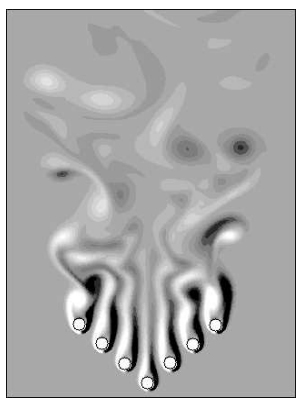

(d)

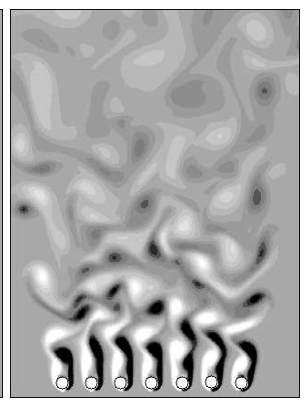

(f)
Figure 10. Vorticity Field for the ' $V$ ' Configuration. $\alpha=40^{\circ}$ (a), $\alpha=60^{\circ}$ (b), $\alpha$ $=80^{\circ}$ (c), $\alpha=100^{\circ}$ (d), $\alpha=140^{\circ}$ (e) and $\alpha=180^{\circ}$ (f).

The drag coefficients and Strouhal numbers for the cylinders 1 , 3,5 and 7 divided by the value of a single cylinder, are presented in Fig. 11. All the cylinders have for $\alpha<100^{0}$ drag coefficients lower than for a single cylinder $\left(C_{d \text { single }}=1.3997, S t_{\text {single }}=0.1955\right)$. As the angle decreases, the wake becomes more inhibited. The amplitude of the wake oscillations increases with $\alpha$. Also, as $\alpha$ increases, the time variation of the drag coefficient becomes more and more complex. Strouhal and drag increase with $\alpha$ for all cylinders and reach values higher than that for an isolated cylinder, for higher angles. These parameters are influenced by the number of cylinders and the angle of the configuration. These simulations can be explored of different ways by changing the Reynolds, the number of cylinders, the angle of the configuration or the diagonal distance between the cylinders. 


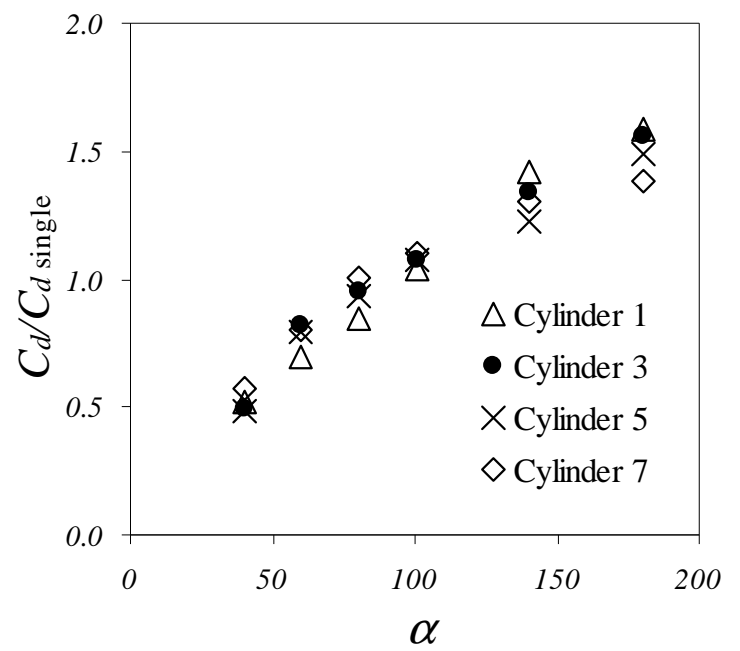

(a)

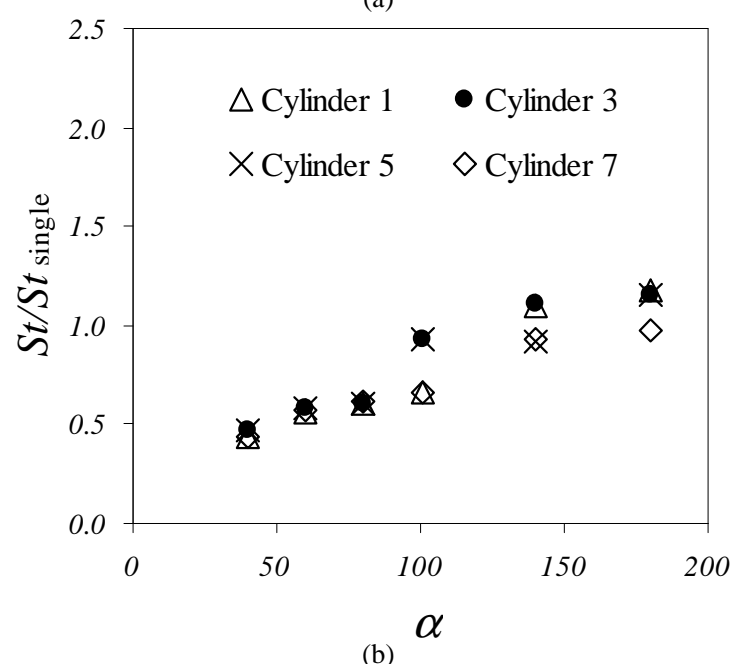

Figure 11. Drag Coefficient ratio (a) and Strouhal number ratio (b) as function of $\alpha$ for the ' $V$ ' Configuration. $C_{d \text { single }}$ and $S t_{\text {single }}$ is the drag and Strouhal of a single cylinder.

\section{Combination of Bluff Bodies of Several Shapes}

The IB methodology with the VPM has the advantage of providing a flow simulation around several geometries, without any type of additional complexity in the grid generation process. The grid is always Cartesian irrespective of the complexity of the configuration of immersed bodies. It is possible to obtain the force coefficients on each immersed body or an overall value, because the force distribution is known over each body individually at each time step.

In order to illustrate these characteristics, a transverse horizontal cut at the main tower of an offshore structure was idealized. These structures are subject to strong wind streams and maritime currents, which can cause vibration, damaging the structural integrity. The schematic view of a composition of bluff bodies, generated by the transverse cut, is shown in Fig. 12(a).

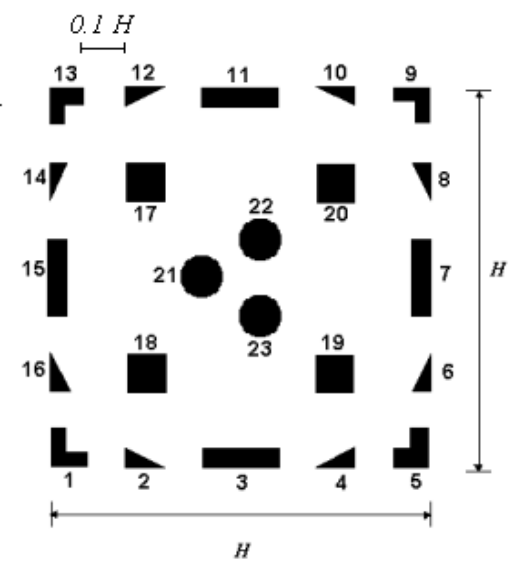

(a)

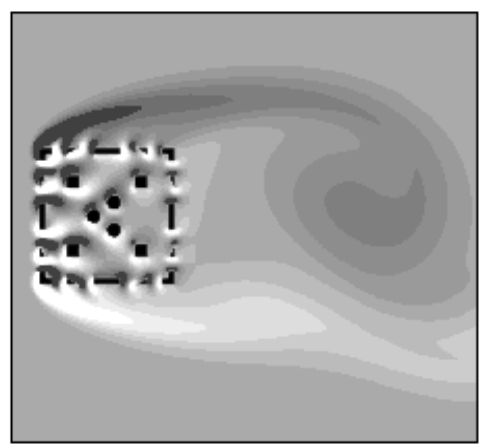

(b)

Figure 12. Schematic view of a horizontal cut in a central tower of an offshore structure (a) and instantaneous vorticity field (b).

Each body was numbered to facilitate the identification. For these simulations a non-uniform grid with $400 \times 300$ points was used The Reynolds number based on the dimension $H$, Fig. 12(a), is $\operatorname{Re}=$ 200. The distance between the external bodies (bodies 1 to 16) was chosen equal to $0.1 \mathrm{H}$, as indicated in Fig. 12(a).

The results showed that the fluid flows through the immersed bodies but there is no vortex shedding inside the structure because all bodies are very close, as can be seen in Fig. 12(b) that presents the instantaneous vorticity field. The system of bodies can be considered as a porous media. The recirculation bubble generated behind the global structure has the same order of $H$ and the flow is unsteady. It could be observed that there was mass injection in the shear layer due to the gap between the bodies. As a consequence the horizontal length of the recirculation bubble was bigger than that of the flow over a square of the same dimensions. The value of the global drag coefficient calculated by the sum of the forces from each body is equal to 1.9492 . The mean drag coefficient of the square is 1.6844. This value is $13 \%$ smaller than the drag coefficient of the composition of bluff bodies.

This results illustrate that it is possible, using the IB-VPM methodology to easily calculate the drag and lift coefficients of each body that compose such a complex geometry.

\section{Conclusions}

The Immersed Boundary Method with the Virtual Physical Model was used to simulate flows over different compositions of bluff bodies. The results of the mean drag coefficient and the Strouhal number for cylinders in tandem and side-by-side were compared with other author's results. The flow dynamics changes with the distance between the cylinders. It was observed the 
presence of a repulsive force when the distance between the bodies is small. Consequently the drag coefficient is affected and can change from negative to positive. For the simulations with different cylinders and with two identical cylinders in tandem, it was observed that the distance where the $C_{d}$ sign changes was $2.7 d$ and $4.0 d$, respectively. In the side-by-side arrangement, a repulsive force between the cylinders has been observed for gaps $L \leq 2.0 \mathrm{~d}$. The simulations with seven cylinders in a ' $\mathrm{V}$ ' configuration show the potential of the methodology to study flows over complex geometries. There is no restriction on the number of bodies and the computational cost is not significantly increased when a great number of bodies are immersed in the flow. In other words the main cost is due to the solution of the linear system for the pressure correction. With these simulations, it is possible to choose the optimal configuration for which the drag coefficients are minimized. The last case simulated was the flow in the presence of different bluff bodies, illustrating a horizontal section on an offshore structure. The global drag coefficient was compared with that of a square with the same dimensions. The drag coefficient value obtained for the compact square was approximately $13 \%$ smaller than that for the composed geometry. There was a change in the shape of the recirculation bubble and the vortex shedding starts earlier than it is for the simulations with a square. It is also important to emphasize that in the IB method a Cartesian grid is used to simulate flows over any geometric shape. Another advantage is the ease of calculating the force coefficients for an isolated body or the global value for the configuration.

\section{Acknowledgements}

The authors acknowledge FAPEMIG (Foundation of Support to the Research of the State of Minas Gerais, Brazil - TEC 00084/06), CNPq (National Council for Scientific and Technological Development, from Brazilian Ministry of Education, Brazil), the Mechanical Engineering College of the Federal University of Uberlândia-MG and the Institute of Mechanical Engineering of the Federal University of Itajubá-MG.

\section{References}

Armfield S. and Street R, 1999, "The fractional-step method for the navier-stokes equations on staggered grids: The accuracy of three variations", Journal of Computational Physics, Vol. 153, pp. 660-665.

Bearman P.W. and Wadcock A.J., 1973, "The interaction between a pair of circular cylinders normal to a stream", Journal Fluid Mechanics, Vol. 61, pp. 499-511.
Fadlun E.A., Verzicco R., Orlandi P. and Mohd-Yusof J., 2000, "Combined immersed-boundary finite-difference methods for threedimensional complex flow simulations", Journal of Computational Physics, Vol. 161, pp. 35-60.

Flatschart R.B., Saltara F., Ferrari Jr J.A., Meneghini J.R. and Siqueira C.R., 2000, "Numerical simulation of flow interference between two circular cylinders in tandem", $19^{\text {th }}$ International Conference on Offshore Mechanics and Artic Engineering, February 14-17, New Orleans, LA, USA.

Goldstein D., Adachi T. and Sakata H., 1993, "Modeling a no-slip flow with an external force field", Journal of Computational Physics, Vol. 105, pp. 354-366, doi: 10.1006/jcph.1993.1081.

Griffth B.E., Hornung R.D., McQueen D.M. and Peskin C.S., 2006, "An adaptive, formally second order accurate version of the immersed boundary method", Journal of Computational Physics, doi:10.1016/j.jcp.2006.08.019.

Kim J., Kim D. and Choi H., 2001, "An immersed-boundary finitevolume method for simulations of flow in complex geometries", Journal of Computational Physics, Vol. 171, pp. 132-150, doi:10.1006/jcph.2001.6778.

Lima e Silva A.L.F., Silveira-Neto A. and Damasceno J.J.R., 2003, "Numerical simulation of two dimensional flows over a circular cylinder using the immersed boundary method", Journal of Computational Physics, Vol. 189, pp. 351-370, doi: 10.1016/S0021-9991(03)00214-6.

Meneghini J.R.., Saltara F., Siqueira C.L.R. and Ferrari Jr J.A., 2001, "Numerical simulation of flow interference between two circular cylinders in tandem and side-by-side arrangements", Journal Fluids and Structures, Vol. 15, pp. 327-350, doi:10.1006/jfls.2000.0343.

Miller L.A. and Peskin C.S., 2005, "A computational fluid dynamics of 'clap and fling' in the smallest insects", The Journal of Experimental Biology, Vol. 208, pp. 195-212, doi:10.1242/jeb.01376.

Mohd-Yusof J., 1997, "Combined immersed boundaries/B-splines methods for simulations of flows in complex geometries", CTR Annual Research Briefs in NASA Ames Research center//Stanford University Center for Turbulence Research, pp. 317-327.

Peskin C.S., 1977, "Numerical analysis of blood flow in the heart", Journal of Computational Physics, Vol. 25, pp. 220-252.

Saiki E.M. and Biringen S., 1996, "Numerical simulation of a cylinder in uniform flow: Application of a virtual boundary method", Journal of Computational Physics, Vol. 123, pp. 450-465.

Schneider G.E. and Zedan M., 1981, "A modified strongly implicit procedure for the numerical solution of field problems", Numerical Heat Transfer, Vol. 4, pp. 1-19.

Surmas R., Santos L.O.E. and Philippi P.C., 2003, "Características da formação e desprendimento de vórtices em grupos de cilindros", II Congresso Brasileiro de Pesquisa e Desenvolvimento em Petróleo e Gás. (In Portuguese)

Ye T., Mittal R., Udaykumar H.S. and Shyy W., 1999, "An accurate cartesian grid method for viscous incompressible flows with complex immersed boundaries", Journal of Computational Physics, Vol. 156, pp. 209-240.

Williamson C.H.K., 1985, "Evolution of a single wake behind a pair of bluff bodies", Jounal Fluid Mechanics, Vol. 159, pp. 1-18. 